\title{
Contributing Authors
}

\author{
Efthymia Alexopoulou \\ CRES, Center for Renewable Energy Sources \\ Biomass Department \\ 19th Km Marathonos Avenue, Pikermi Attikis, \\ Greece \\ Chapter 3
}

\section{Prince N. Amaniampong}

Institut de Chimie des Milieux et Matériaux de Poitiers, Université de Poitiers, ENSIP, 1 rue Marcel Doré, Poitiers, France prince.nana.amaniampong@univ-poitiers.fr Chapter 13

\section{Claudia Antonetti and Industrial Chemistry, Via Risorgimento 35, Pisa, Italy claudia.antonetti@unipi.it \\ Chapter 6}

University of Pisa, Department of Chemistry

\section{Michele Aresta}

$I^{2} R$ Ltd, Tecnopolis, Valenzano, Italy michele.aresta@ic2r.com

Chapters 1, 12

\section{Catia Bastioli} NOVAMONT S.p.A., Via Fauser 8, Novara, Italy catia.bastioli@novamont.com

Chapters 2, 17

\section{Daniele Bianchi}

Eni S.p.A.; Technology, R\&D \& Digital; Research \& Technological Innovation. Via Fauser 4, Novara, Italy daniele.bianchi2@eni.com Chapter 11

\author{
Simone Cailotto \\ Department of Molecular Sciences and \\ Nanosystems, Ca' Foscari University of \\ Venice, \\ Via Torino 155, Venezia Mestre, Italy \\ Chapter 9
}

\section{Christos Christodoulou}

Center for research and technology Hellas

Arkat Athens, Greece

Chapter 10

\section{Myrsini Christou}

CRES, Center for Renewable Energy Sources Biomass Department

19th Km Marathonos Avenue, Pikermi Attikis,

Greece

mchrist@cres.gr

Chapter 3

\section{Claudia Crestini}

Department of Molecular Sciences and Nanosystems, Ca' Foscari University of Venice, Via Torino 155, 30170 Venezia Mestre, Italy

claudia.crestini@unive.it

Chapter 9

\section{Jasmina Cziommer}

Department of Biotechnology, Technische

Universität,

Berlin, Ackerstraße 76 ACK24, Berlin,

Germany

Chapter 8

\section{Christophe Darcel}

Sciences Chimiques de Rennes, Université de Rennes,

263 Avenue Général Leclerc, Rennes, France christophe.darcel@univ-rennes1.fr

Chapter 13 


\section{Karine De Oliveira Vigier}

Institut de Chimie des Milieux et Matériaux de Poitiers, Université de Poitiers, ENSIP, 1 rue Marcel Doré, Poitiers, France karine.vigier@univ-poitiers.fr Chapter 13

\section{Angela Dibenedetto}

Department of Chemistry, University of Bari, Campus Universitario and CIRCC via Celso Ulpiani 26, Bari, Italy angela.dibenedetto@uniba.it Chapters 1, 4, 14, 15

\section{Franck Dumeignil}

Univ. Lille, UCCS, UMR CNRS 8181, 59655 Villeneuve-d'Ascq Cedex, France Chapter 1

\section{loannis Eleftheriadis}

CRES, Center for Renewable Energy Sources Biomass Department

19th Km Marathonos Avenue, Pikermi Attikis, Greece,

Chapter 3

\section{Hegon Euson}

Univ. Lille, Université de Liège, UMRT 1158

BioEcoAgro - Biotransformation/ Enzymes et Biocatalyse, Lille, France

egon.heuson@univ-lille.fr

Chapter 15

\section{Cédric Fischmeister}

Sciences Chimiques de Rennes, Université de Rennes,

263 Avenue Général Leclerc, Rennes, France cedric.fischmeister@univ-rennes1.fr Chapter 13

\section{Michèle Friend}

Univ. Lille, CNRS, Centrale Lille, Univ. Artois, UCCS - Unité de Catalyse et Chimie du Solide, Lille, France, \& George Washington University, Washington D.C., USA

michele@gwu.edu

Chapter 18

\section{Anna Maria Raspolli Galletti}

University of Pisa, Department of Chemistry and Industrial Chemistry, Via Risorgimento 35, Pisa, Italy, anna.maria.raspolli.galletti@unipi.it

Chapter 6

\section{Maria Georgiadou}

European Commission, Directorate-General for Research \& Innovation Unit Clean Energy Transition Rue Champ de Mars 21, Brussels, Belgium maria.georgiadou@ec-europa.eu Introduction

\section{Cecilia Giardi} NOVAMONT S.p.A., Via Fauser 8, Novara, Italy cecilia.giardi@novamont.com Chapter 2

\section{Matteo Gigli}

Department of Molecular Sciences and Nanosystems, Ca' Foscari University of Venice, Via Torino 155, Venezia Mestre, Italy Chapter 9

\section{François Jérôme}

Institut de Chimie des Milieux et Matériaux de Poitiers, Université de Poitiers, ENSIP, 1 rue Marcel Doré, Poitiers, France francois.jerome@univ-poitiers.fr Chapter 13

\section{Stefan Junne}

Department of Biotechnology, Technische Universität, Berlin, Ackerstraße 76 ACK24, Berlin, Germany stefan.junne@tu-berlin.de Chapter 8

\section{Efthymia-loanna Koytsoumpa} Center for research and technology Hellas Arkat Athens, Greece Chapter 10 


\section{Michal Krzyzaniak}

UWM, University of Warmia and Mazury in Olsztyn, Department of Plant Breeding and Seed Production

Plac Łódzki 3/420, Olsztyn, Poland

Chapter 3

\section{Loïc Leclercq}

Université de Lille, Cité Scientifique, Villeneuve d'Ascq, France

loic.leclercq@univ-lille.fr

Chapter 16

\section{Gabriel Morales}

Department of Chemical and Environmental Technology, ESCET,

Universidad Rey Juan Carlos, Móstoles, Spain Chapter 7

\section{Juan A. Melero}

Department of Chemical and Environmental Technology, ESCET,

Universidad Rey Juan Carlos, Móstoles, Spain Chapter 7

\section{Véronique Nardello-Rataj}

Centrale Lille Institut, Cité Scientifique, Villeneuve d'Ascq, France veronique.rataj-nardello@univ-lille.fr Chapter 16

\section{Peter Naubauer}

Department of Biotechnology, Technische Universität, Berlin, Ackerstraße 76 ACK24, Berlin, Germany Chapter 8

\section{Francesco Nocito}

Department of Chemistry, University of Bari, Campus Universitario, and CIRCC, via Celso Ulpiani 26, Bari, Italy

francesco.nocito@uniba.it

Chapter 14

\section{Marta Paniagua}

Department of Chemical, Energy and Mechanical Technology, ESCET, Universidad Rey Juan Carlos, Móstoles, Spain Chapter 7

\section{Kyriakos D. Panopoulos}

Center for research and technology Hellas Arkat Athens, Greece. panopoulos@certh.gr Chapter 10

\section{Ioanna Papamichael}

CRES, Center for Renewable Energy Sources Biomass Department

19th Km Marathonos Avenue, Pikermi Attikis, Greece

Chapter 3

\section{Carlo Perego}

Via Ss. Cornelio e Cipriano 15/E, Carnate (MB), Italy.

caregoperlo54@gmail.com

Chapter 11

\section{Christin Prescher}

Institute of Bioprocess and Biosystems

Engineering,

Hamburg University of Technology, Hamburg, Germany

christin.prescher@web.de

Chapter 5

\section{Marco Ricci}

via Fra Dolcino 19, Novara, Italy

riccimontironi@libero.it

Chapter 11

\section{Wael Sabra}

Institute of Bioprocess and Biosystems

Engineering, Hamburg University of Technology, Hamburg, and Department of Life Sciences, Rhein-Waal-University of Applied Sciences, Kleve, Germany wael.sabra@hochschule-rhein-waal.de Chapter 5

\section{Fabio Sagnelli} NOVAMONT S.p.A., Via Fauser 8, Novara, Italy fabio.sagnelli@novamont.com Chapter 2 
Patrizio Salice

NOVAMONT S.p.A.,

via Fauser, 8 - Novara, Italy

patrizio.salice@novamont.com

Chapter 17

\section{Ludwig Selder}

Institute of Bioprocess and Biosystems

Engineering,

Hamburg University of Technology, Hamburg,

Germany

ludwig.selder@tuhh.de

Chapter 5

\section{David P. Serrano}

Thermochemical Processes Unit, IMDEA

Energy Institute, and Department of

Chemical, Energy and Mechanical

Technology, ESCET, Universidad Rey Juan

Carlos,

Móstoles, Spain

david.serrano@imdea.org

Chapter 7

\section{Mariusz Stolarski}

UWM, University of Warmia and Mazury in Olsztyn, Faculty of Environmental

Management and Agriculture, Department of

Plant Breeding and Seed Production

Plac tódzki 3/420, Olsztyn, Poland

Chapter 3

\section{Simon Tauber}

Department of Biotechnology, Technische

Universität, Berlin,

Ackerstraße 76 ACK24, Berlin, Germany

Chapter 8

\section{Charles Themistocles}

SOABE, Société Agricole de Befandriana-Sud

\& Partners Sarl,

Antananarivo 101, Madagascar

Chapter 3

\section{Kostas Tsiotas}

CRES, Center for Renewable Energy Sources Biomass Department

19th Km Marathonos Avenue, Pikermi Attikis, Greece

Chapter 3

\section{Wei Wang}

Institute of Bioprocess and Biosystems

Engineering,

Hamburg University of Technology, Hamburg,

Germany

wei.wang@tuhh.de

Chapter 5

\section{An-Ping Zeng}

Institute of Bioprocess and Biosystems

Engineering,

Hamburg University of Technology, Hamburg, Germany

aze@tuhh.de

Chapter 5 